\title{
Easy axis magnetization reversal in cobalt antidot arrays
}

\author{
E. Mengotti, L. J. Heyderman, ${ }^{\text {a) }}$ and F. Nolting \\ Paul Scherrer Institut, 5232 Villigen PSI, Switzerland \\ B. R. Craig ${ }^{\text {b) }}$ and J. N. Chapman \\ Department of Physics and Astronomy, University of Glasgow, Glasgow G12 8QQ, United Kingdom \\ L. Lopez-Diaz \\ Departamento Fisica Aplicada, Universidad de Salamanca, Plaza de la Merced, Salamanca 37008, Spain \\ R. J. Matelon ${ }^{\text {c) }}$ and U. G. Volkmann \\ Facultad de Física, Pontificia Universidad Católica de Chile, Santiago 22, Chile \\ M. Kläui and U. Rüdiger \\ Fachbereich Physik, Universität Konstanz, 78457 Konstanz, Germany \\ C. A. F. Vaz ${ }^{\text {d) }}$ and J. A. C. Bland \\ Cavendish Laboratory, University of Cambridge, Madingley Road, Cambridge CB3 OHE, United Kingdom
}

(Presented on 7 November 2007; received 5 September 2007; accepted 27 September 2007; published online 23 January 2008)

\begin{abstract}
The magnetization reversal in square lattice cobalt antidot arrays with the applied field at $45^{\circ}$ to the antidot rows was investigated using Lorentz electron microscopy in the Fresnel mode. While the hysteresis loops from magneto-optical Kerr effect measurements only reflect the easy axis character of the reversal, several different reversal processes were identified in the Fresnel images depending on the field history. Details of this complex magnetization reversal were elucidated with micromagnetic simulations. (C) 2008 American Institute of Physics. [DOI: 10.1063/1.2829006]
\end{abstract}

When a regular array of holes is introduced into a continuous ferromagnetic thin film, the magnetic properties are significantly changed. ${ }^{1-5}$ In such ferromagnetic antidot arrays, domain wall behavior and interactions can be investigated, topics which are currently of interest for future magnetic devices based on the domain walls. ${ }^{6,7}$ The basic domain configuration for square lattice antidot arrays with antidot size $\approx$ antidot separation is shown in the schematic diagrams 1 to 3 in Fig. 3. This configuration results from the fact that the spins adjacent to the antidots align parallel to the antidot borders in order to reduce the stray field energy and is observed with photoemission electron microscopy (PEEM) as a periodic checked domain contrast commensurate with the antidot lattice. ${ }^{8}$ The hysteresis loops with the applied field along the hard and easy axes, i.e., parallel and at $45^{\circ}$ to the antidot rows (see schematic diagram in Fig. 1), have been measured and the basic domain states have been observed. ${ }^{1,5}$ However, very little is known about the details of magnetization reversal processes. Recently, we showed that on application of an in-plane magnetic field parallel to the antidot columns, magnetization reversal occurs by nucleation and propagation of chains of magnetic domains which have discrete lengths corresponding to multiples of the antidot period. ${ }^{9}$ In addition, when the ends of two orthogonal do-

\footnotetext{
${ }^{a)}$ Electronic mail: laura.heyderman@psi.ch.

${ }^{b)}$ Present address: Seagate Technology, 1 Disc Drive, Springtown Industrial Estate, Derry BT48 0BF, UK.

${ }^{c)}$ Present address: School of Engineering, Computer Science and Mathematics, University of Exeter, EX4 4QF, UK.

${ }^{d)}$ Present address: Applied Physics, Yale University, New Haven, Connecticut 06520 .
}

main chains coincide, a stable domain wall configuration is formed giving a pinning center for propagating domain walls. Depending on the film thickness, this domain wall configuration comprises either a $180^{\circ}$ wall or four $90^{\circ}$ walls forming an antivortex. ${ }^{9,10}$ Here, we report on the magnetization reversal in square lattice cobalt antidot arrays on application of a magnetic field at an angle of $45^{\circ}$ to the antidot rows.

Cobalt antidot arrays were fabricated by electron beam lithography. For observations with Lorentz electron microscopy, it was necessary to place the antidot arrays on substrates which allow the transmission of electrons. We therefore used silicon nitride $\left(\mathrm{Si}_{3} \mathrm{~N}_{4}\right)$ membrane chips with 500 $\times 500 \mu \mathrm{m}^{2}$ membrane windows which are 50-nm-thick (from Silson Ltd., UK). It was not possible to use lift-off for pattern transfer since this method is assisted with an ultrasound bath which will break the fragile membranes. Instead, arrays of holes were created in the $\mathrm{Si}_{3} \mathrm{~N}_{4}$ membranes using electron beam lithography in combination with reactive ion etching (RIE) processes and the cobalt thin film was subsequently deposited on to the prepatterned membranes. ${ }^{10}$ The deposition was carried out by dc-magnetron sputtering [base pressure $\left.=(1.5-5.5) \times 10^{-7} \mathrm{mbar}\right]$ and the cobalt film was capped with a 1-nm-thick aluminum layer to prevent oxidation. Each of the antidot arrays cover a square area with side length of $10-20 \mu \mathrm{m}$, and the antidot period $p$ ranged from $2 \mu \mathrm{m}$ down to $200 \mathrm{~nm}$, with antidot size $\approx$ antidot separation and square or round holes. Due to the limited resolution of the fabrication process, the square holes have rounded corners.

Hysteresis loops were obtained from longitudinal 
(a) $\mathrm{p}=2 \mu \mathrm{m}$

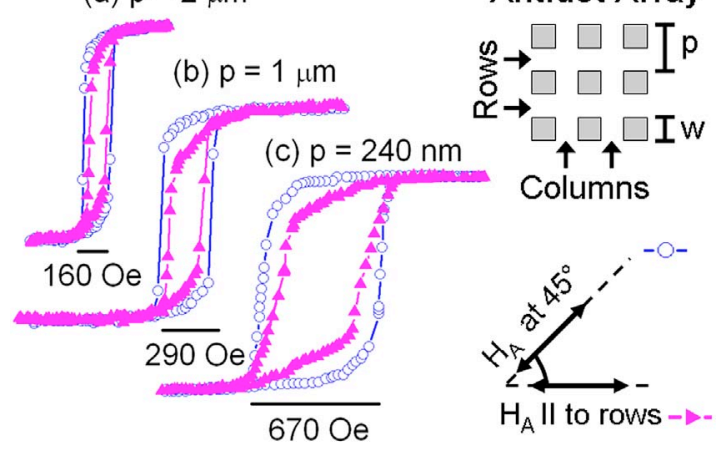

FIG. 1. (Color online) Hysteresis loops obtained from MOKE measurements of antidot arrays with different periods $p$ and magnetic field applied parallel (triangles) and at $45^{\circ}$ to the antidot rows (open circles). The antidot size is $w=p / 2$. A schematic of the antidot array layout and the orientation of applied field $H_{A}$ is given.

magneto-optical Kerr effect (MOKE) measurements on antidot arrays fabricated on bulk silicon substrates as described in Ref. 9. The hysteresis loops obtained for 10-nm-thick Co antidot arrays with periods $p=2 \mu \mathrm{m}, 1 \mu \mathrm{m}$, and $240 \mathrm{~nm}$ are shown in Fig. 1 for two different field orientations: parallel to the antidot rows (triangles), which is equivalent to parallel to the antidot columns, and at $45^{\circ}$ to the antidot rows (open circles). For fields applied along the antidot rows, there is first a small reversible decrease in the magnetization after saturation. Here the spins neighboring the antidots rotate to align themselves parallel to the antidot borders in order to reduce the stray field energy. The second feature of the hysteresis loops is a large change in the magnetization corresponding to the reversal of the antidot array via nucleation and propagation of domain chains. ${ }^{9}$ As the antidot period decreases, both the exchange and magnetostatic contributions increase due to the decreasing lateral dimensions, resulting in the observed increase in the switching field, ${ }^{9}$ i.e., $H_{C}=48 \mathrm{Oe}, 93 \mathrm{Oe}$, and $234 \mathrm{Oe}$ for $p=2 \mu \mathrm{m}, 1 \mu \mathrm{m}$ and $240 \mathrm{~nm}$, respectively. For a field applied at $45^{\circ}$, the loops are squarer and have a higher switching field $\left(H_{C}=80 \mathrm{Oe}\right.$, $145 \mathrm{Oe}$, and $335 \mathrm{Oe}$ for $p=2 \mu \mathrm{m}, 1 \mu \mathrm{m}$, and $240 \mathrm{~nm}$, respectively), displaying the hallmarks of an easy axis reversal. $^{1,5}$

For the magnetic imaging, we employed Lorentz electron microscopy in the Fresnel mode. ${ }^{11}$ This out-of-focus imaging technique reveals the position of domain walls which appear black or white depending on the orientation of the surrounding spins. In order to observe the details of magnetization reversal, an antidot array with $p=800 \mathrm{~nm}$ and square holes was studied. The antidot size is $w=440 \mathrm{~nm}$, giving the ratio $w / p=0.55$. A magnetic field was generated by applying a current to the electron microscope objective lens which gave a vertical field of $\approx 500$ Oe parallel to the electron column. The sample was then tilted in the range of $\pm 20^{\circ}$ to vary the magnitude of the in-plane field $H_{A}$ in the range $\pm 170 \mathrm{Oe}$, and the in-plane field was applied at an angle of $45^{\circ}$ to the antidot rows. Two Fresnel images taken during a single field cycle are shown in Fig. 2. Here $H_{A}$ was first set to $-170 \mathrm{Oe}$, then decreased to zero, and images were recorded as $H_{A}$ was increased in the reverse direction. First, local do-

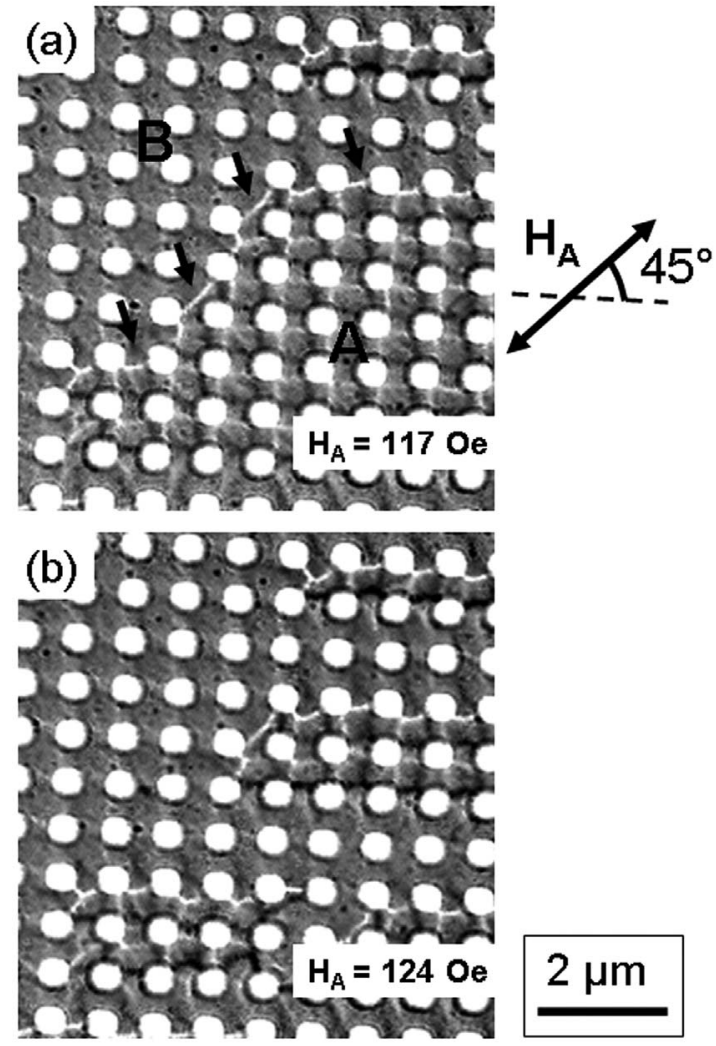

FIG. 2. Magnetic domain configurations observed with Fresnel microscopy in an antidot array, with $p=800 \mathrm{~nm}$ and $w / p=0.55$. An in-plane field $H_{A}$ at $45^{\circ}$ to the antidot rows was decreased from -170 Oe to zero and then increased to (a) $117 \mathrm{Oe}$ and (b) $124 \mathrm{Oe}$. The global boundary in (a), indicated by the arrows, separates a region with high magnetic contrast (A) corresponding to the basic antidot configuration and a reversed region with low contrast (B) indicating parallel spins. After the global boundary has passed through the sample, horizontal domain chains are left behind (b)

main walls form between the antidots as the spins relax to the basic antidot configuration ${ }^{9}$ consisting of small square domains as seen in the region denoted by A in Fig. 2(a). Then a long domain wall, or global boundary indicated by the arrows in Fig. 2(a), moves through the antidot array. This global boundary separates region A with high magnetic contrast from the region denoted by $\mathrm{B}$ where magnetization reversal has occurred. In region $\mathrm{B}$, the reduced magnetic contrast indicates that the spins are more or less parallel and are likely to point in or close to the field direction. As the global boundary continues to pass through the antidot array, the reversed region increases and horizontal domain chains are left behind [Fig. 2(b)]. The domain chains finally disappear at $H_{A}=151 \mathrm{Oe}$ and the magnetization saturates. A similar reversal behavior was seen when the magnetic field was applied in the reverse direction, except that the chains were vertical rather than horizontal. We also observed the magnetization reversal for different orientations of applied field with respect to the antidot rows from $42^{\circ}$ to $48^{\circ}$ in $1^{\circ}$ steps. Again, we observed rotation of the spins in local domains, motion of global domain boundaries, and formation of chains, either parallel or perpendicular to the antidot rows, with differences in behavior depending on whether the field was applied in the positive or negative direction but no obvious trend as a function of field angle. 

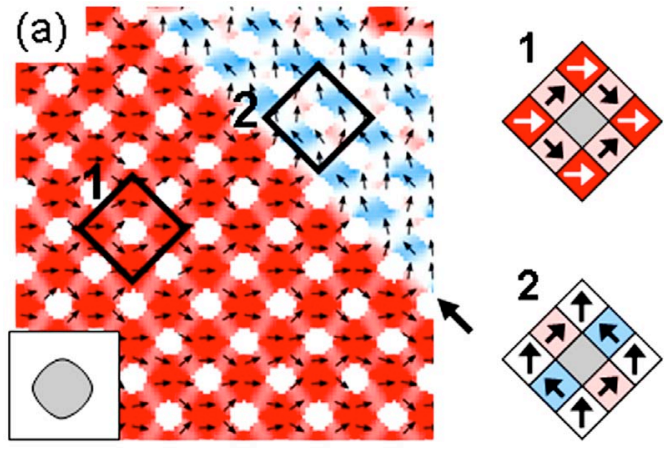

(b)

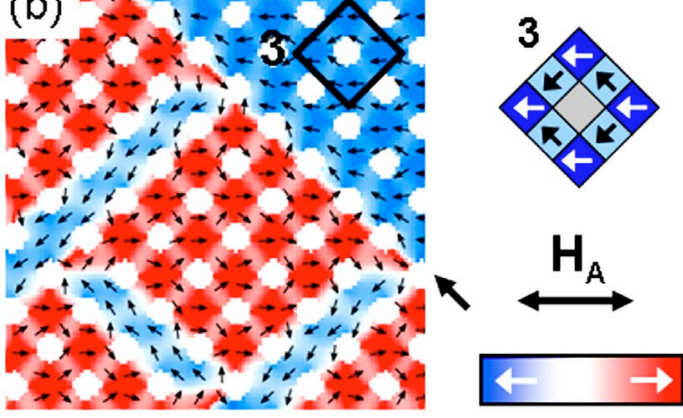

FIG. 3. (Color online) Two snapshots of a micromagnetic simulation for an antidot array with $p=200 \mathrm{~nm}$ and $w / p=0.55$. The applied field $H_{A}$ was decreased from +500 Oe down to $-1000 \mathrm{Oe}$, and the snapshots are for (a) $H_{A}=-143$ Oe and (b) $H_{A}=-520$ Oe. The three differently oriented leaf configurations which occur are shown schematically and the global boundary is indicated in each snapshot with an arrow. The hole shape for the antidot array imaged in Fig. 2 is used in the simulation [inset of Fig. 3(a)].

To elucidate this complex reversal behavior, micromagnetic simulations were performed using OOMMF on a 10-nm-thick cobalt film containing a square lattice antidot array with an area $1.9 \times 1.9 \mu \mathrm{m}^{2}, p=200 \mathrm{~nm}$, and using a simulation cell size of $5 \times 5 \mathrm{~nm}^{2}$. The array was composed of square antidots with $w / p=0.55$ and rounded corners to mimic the shape of the holes in the real array [see inset in Fig. 3(a)]. Typical parameters for cobalt were chosen for the simulation: saturation magnetization $M_{s}=1400 \times 10^{3} \mathrm{~A} / \mathrm{m}$, exchange constant $A=3 \times 10^{-11} \mathrm{~J} / \mathrm{m}$, and uniaxial anisotropy constant $K_{U}=3.5 \times 10^{3} \mathrm{~J} / \mathrm{m}^{3}$. The external field was applied at $45^{\circ}$ to the antidot rows and was decreased from $+500 \mathrm{Oe}$ down to -1000 Oe in 1500 steps. Two snapshots of the simulated magnetization reversal process are shown in Fig. 3. First, there is a relaxation to the basic antidot leaf configuration $^{9}$ (configuration 1 in Fig. 3). Then, in a region towards the top right corner of the array [Fig. 3(a)], the spins pointing along the antidot rows (running top left to bottom right) reverse resulting in a leaf configuration which is now rotated by $90^{\circ}$ shown as configuration 2 in Fig. 3. There is a global boundary between the regions containing configurations 1 and 2 (indicated by the arrow) whose position does not change with increasing applied field. Instead, on increasing $H_{A}$ to $-500 \mathrm{Oe}$, in the top right region the spins pointing along the antidot columns (running bottom left to top right) reverse resulting in a leaf configuration which is again rotated by $90^{\circ}$ shown as configuration 3 in Fig. 3. On further increase of the field, instead of expansion of this region, the magnetization reversal continues by nucleation and propagation of chains of domains along the antidot rows and columns [Fig. 3(b)]. Finally, the magnetization saturates at $H_{A}$ $=-700$ Oe, following the annihilation of a few short $360^{\circ}$ domain walls between adjacent antidots (not shown).

Several different simulations were performed, varying $w / p$ from 0.45 to 0.65 and using various angles of the field to the antidot rows between $45^{\circ}$ and $48^{\circ}$. In addition, a $z$-field component was added $\left(H_{z}=500 \mathrm{Oe}\right)$ to better reproduce the experiment and the period was increased from 200 to $400 \mathrm{~nm}$. For all of these cases, no significant change in the reversal processes was found.

In the Fresnel images several processes were identified: local rotation of the magnetization between the antidots, and formation of global boundaries and domain chains. The balance of the various processes during reversal and the orientation of the domain chains is dependent on the field history, i.e., the field orientation and direction (positive or negative). While there are domain chains of width $p / 2$ present in both the Lorentz microscopy measurements and the micromagnetic simulations, there is a difference in the way that they are formed. This can be attributed to, for example, the presence of defects which facilitate nucleation, array size differences, and thermal effects in the real antidot arrays. The understanding of the complexity of the magnetization reversal processes in antidot arrays gained here will help the interpretation of macroscopic measurements, such as hysteresis loop and magnetoresistance measurements, particularly when the antidot period is reduced below the resolution of available imaging techniques.

The authors would like to thank at the Paul Scherrer Institut, Michael Horisberger for the material deposition, and Eugen Deckardt, Anja Weber, and Christian David for their support with electron beam lithography. Support by the Swiss SNF, EPSRC (UK) and DFG (SFB 513) is gratefully acknowledged.

${ }^{1}$ C. T. Yu, H. Jiang, L. Shen, P. J. Flanders, and G. J. Mankey, J. Appl. Phys. 87, 6322 (2000).

${ }^{2}$ A. O. Adeyeye, J. A. C. Bland, and C. Daboo, Appl. Phys. Lett. 70, 3164 (1997).

${ }^{3}$ C. C. Wang, A. O. Adeyeye, and Y. H. Wu, J. Appl. Phys. 94, 6644 (2003). ${ }^{4}$ I. Guedes, M. Grimsditch, V. Metlushko, P. Vavassori, R. Camley, B. Ilic, P. Neuzil, and R. Kumar, Phys. Rev. B 67, 024428 (2003).

${ }^{5}$ P. Vavassori, G. Gubbiotti, G. Zangari, C. T. Yu, H. Yin, H. Jiang, and G. J. Mankey, J. Appl. Phys. 91, 7992 (2002).

${ }^{6}$ D. A. Allwood, G. Xiong, C. C. Faulkner, D. Atkinson, D. Petit, and R. P. Cowburn, Science 309, 1688 (2005)

${ }^{7}$ M. Hara, J. Shibata, T. Kimura, and Y. Otani, Appl. Phys. Lett. 89, 192504 (2006)

${ }^{8}$ L. J. Heyderman, F. Nolting, and C. Quitmann, Appl. Phys. Lett. 83, 1797 (2003).

${ }^{9}$ L. J. Heyderman, F. Nolting, D. Backes, S. Czekaj, L. Lopez-Diaz, M. Klaui, U. Rudiger, C. A. F. Vaz, J. A. C. Bland, R. J. Matelon, U. G. Volkmann, and P. Fischer, Phys. Rev. B 73, 214429 (2006).

${ }^{10}$ L. J. Heyderman, S. Czekaj, F. Nolting, D.-H. Kim, and P. Fischer, J. Magn. Magn. Mater. 316, 99 (2007).

${ }^{11}$ J. N. Chapman, R. P. Ferrier, L. J. Heyderman, S. McVitie, W. A. P. Nicholson, and B. Bormans, Electron Microscopy and Analysis (IOP Publishing Ltd., Bristol, 1993), pp. 1-8. 